\title{
Performance Demonstration of Self-Powered Neutron Detectors for Steady-State Reactor Operations
}

Milestone Report-M2CT-21IN07020213

Kevin Tsai

Idaho National Laboratory

Idaho National Laboratory 


\section{DISCLAIMER}

This information was prepared as an account of work sponsored by an agency of the U.S. Government. Neither the U.S. Government nor any agency thereof, nor any of their employees, makes any warranty, expressed or implied, or assumes any legal liability or responsibility for the accuracy, completeness, or usefulness, of any information, apparatus, product, or process disclosed, or represents that its use would not infringe privately owned rights. References herein to any specific commercial product, process, or service by trade name, trade mark, manufacturer, or otherwise, does not necessarily constitute or imply its endorsement, recommendation, or favoring by the U.S. Government or any agency thereof. The views and opinions of authors expressed herein do not necessarily state or reflect those of the U.S. Government or any agency thereof. 
INL/EXT-21-62961

Revision 0

\title{
Performance Demonstration of Self-Powered Neutron Detectors for Steady-State Reactor Operations
}

\author{
Milestone Report-M2CT-21IN0702013
}

Kevin Tsai

Idaho National Laboratory

May 2021

Idaho National Laboratory

Idaho Falls, Idaho 83415

http://www.inl.gov

Prepared for the U.S. Department of Energy Office of Nuclear Energy Under DOE Idaho Operations Office

Contract DE-AC07-05ID14517 
Page intentionally left blank 


\begin{abstract}
The irradiation testing of sensors in reactors is a crucial step towards calibrating and qualifying sensors prior to their deployment in experiments. This report details the process toward qualifying and calibrating custom-designed rhodium-based self-power neutron detectors (Rh-SPNDs) for steady-state reactor irradiations. This process serves to both demonstrate the performance capabilities of Rh-SPNDs as well as to provide experimental data for development of a sensor sensitivity model.

Two designs of Rh-SPNDs were tested in various reactors to demonstrate: detection resolution in a low neutron flux environment, a delayed-response compensation technique, output linearity in a large range of neutron flux, and measurement accuracy verified with dosimetry. The detection resolution and compensation technique was demonstrated in the AGN-201m reactor at Idaho State University. The irradiation confirmed the sensors' capability to perform steady-state operations in a low neutron flux of $\sim 2 \mathrm{E} 8 \mathrm{n} / \mathrm{cm}^{2}$-sec. Sensor output linearity coupled with the delayed-response compensation was investigated at the neutron radiography reactor at Idaho National Laboratory. A Rh-SPND was irradiated to neutron fluxes ranging from $2 \mathrm{E} 8$ to $2 \mathrm{E} 13 \mathrm{n} / \mathrm{cm}^{2}-\mathrm{sec}$ range. The measured data demonstrated a wide and linear range of operation with a measured linear sensitivity of $1.0129 \times 10^{-13} \mathrm{~A} / \mathrm{W}$ with a correlationsquared value of $r^{2}=0.9927$. The measurement accuracy was investigated at the Advanced Test Reactor Critical reactor. The SPNDs were inserted into a test vehicle with collocated flux wires. Two irradiations with different flux levels were performed, and the SPNDs relative measurement between the two irradiations was calculated to be $1.2613 \pm 0.0153$ for the small SPND design and $1.1809 \pm 0.0108$ for the large SPND design. Both SPND measurements fell between the co-axial dosimetry result, which reported $1.218 \pm 0.047$.
\end{abstract}

Additionally, the preliminary MCNP model for calculating SPND sensitivity was developed in parallel to this work. Modeled neutron spectrum with measured magnitude was used for inputs to determine the simulated SPND output. The results showed an overestimation of signal strength by a factor of 5, which was expected because of model simplification. This leads to future modeling work to account for signal losses from additional physical properties, including high temperature environments for FY-21. 
Page intentionally left blank 


\section{CONTENTS}

ABSTRACT iii

ACRONYMS viii

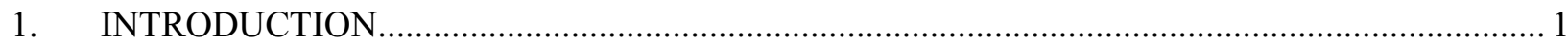

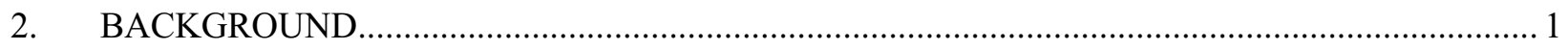

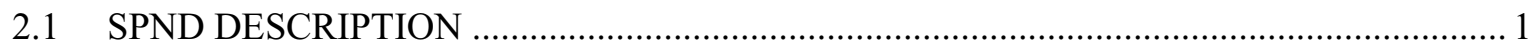

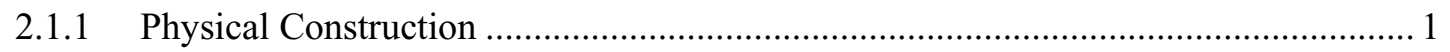

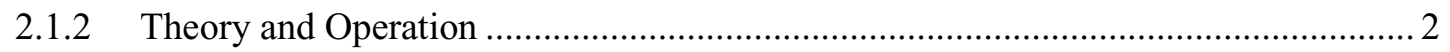

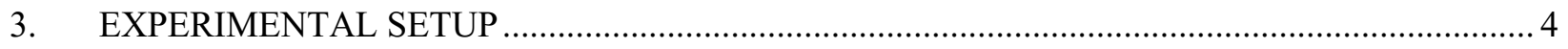

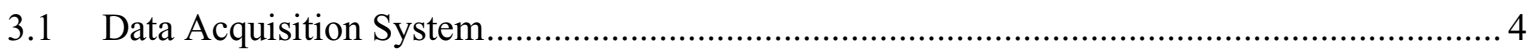

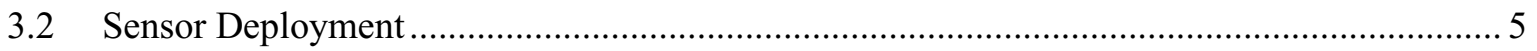

3.2.1 SPND Low-Flux Resolution Testing at AGN-201m ............................................ 5

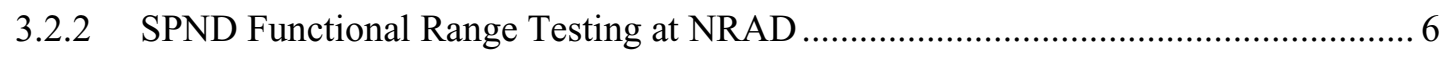

3.2.3 Rh-SPND Deployment for ATRC Medium I-Position Campaign.............................. 7

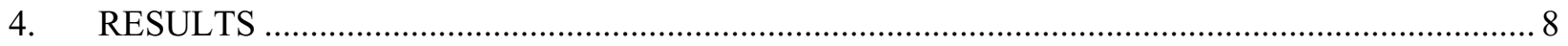

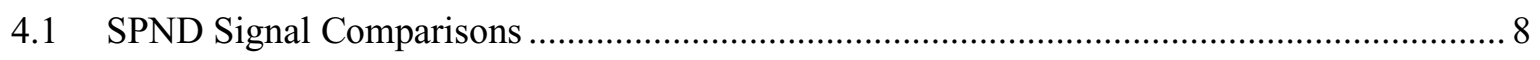

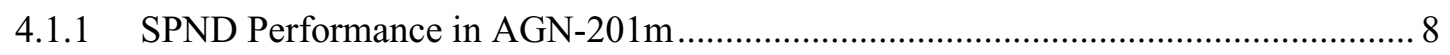

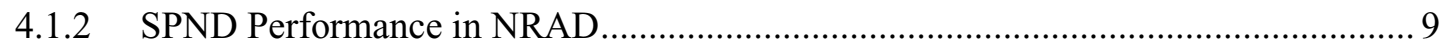

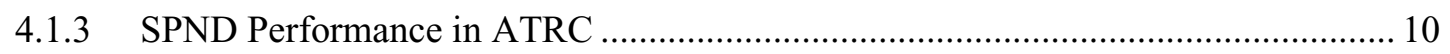

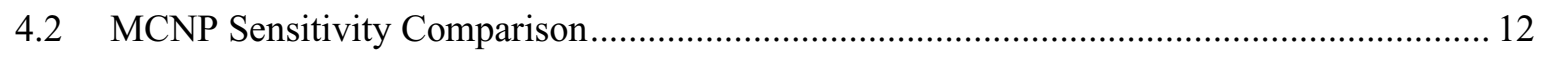

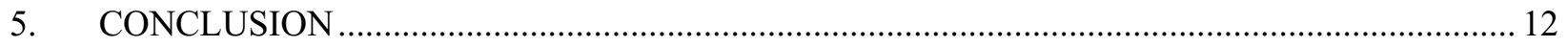

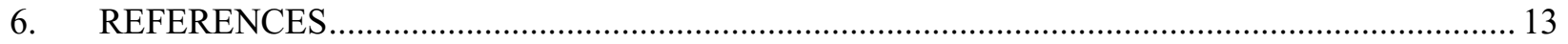

\section{FIGURES}

Figure 1. Conceptual view of a standard SPND design. .....................................................................

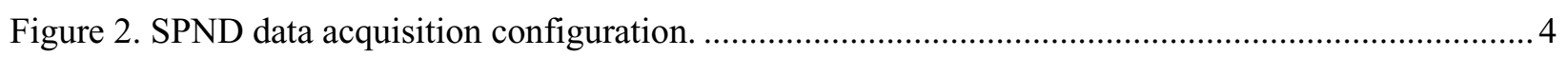

Figure 3. LabVIEW software interface for SPND data recording. ....................................................... 5

Figure 4. (Left) AGN-201m cross-sectional diagram with radiation levels of the reactor operating at 5W. (Right) Insertion of Rh-SPND and Hf-SPND into the core center of AGN-201m reactor.

Figure 5. (Left) NRAD core configuration. (Right) Insertion of Rh-SPND into the F1 irradiation position.

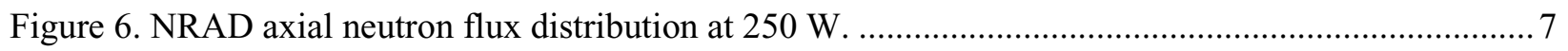

Figure 7. Conceptual image of the booster fuel and beryllium moderator deployed in ATR/ATRC.......... 8

Figure 8. Signal comparison of (left) uncompensated and (right) compensated Rh-SPND with Hf- 
Figure 9. Signal comparison of (left) uncompensated and (right) compensated Rh-SPND with NRAD power.

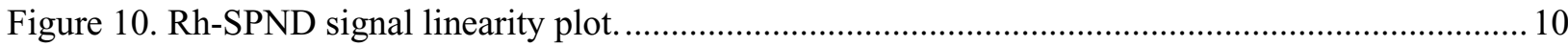

Figure 11. Signal Trace of 080-Rh-SPND and 102-Rh-SPND during ATRC irradiations......................... 11

Figure 12. Comparison of individual Rh-SPND output between irradiations with no booster inserted (NB) and booster inserted (B).

\section{TABLES}

Table 1. SPND materials and geometry comparison between historic Rh-SPND and newly fabricated Rh-SPNDs from Idaho Laboratories Corporation. .................................................... 2

Table 2. Experimental measurement comparison between irradiations without and with booster

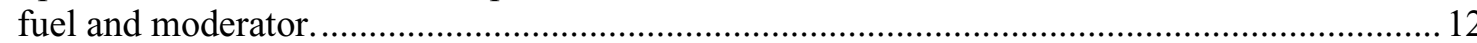

Table 3. Comparison between experimental peak signal vs. MCNP prediction........................................ 12 
Page intentionally left blank 


\section{ACRONYMS}

ATRC Advanced Test Reactor Critical

DAS Data acquisition system

ILC Idaho Laboratories Corporation

INL Idaho National Laboratory

ISU Idaho State University

MIC mineral-insulated cable

NRAD Neutron Radiography

SPND self-powered neutron detector 
Page intentionally left blank 


\section{Performance Demonstration of Self-Powered Neutron Detectors for Steady-State Reactor Operations}

\section{INTRODUCTION}

This report documents the testing of custom-made rhodium-based self-powered neutron detectors (Rh-SPNDs) for the purpose of calibrating and qualifying SPNDs for steady-state irradiation experiments. Complimentary to this effort is the use of SPNDs at the Advanced Test Reactor Critical (ATRC) reactor, a 5kW approximate nuclear mock-up of the Advanced Test Reactor (ATR), in support of the ATR medium I-loop deployment project [1].

A crucial component to the viability of using the ATR medium I-position for advanced fuel testing campaigns is the installment of a booster fuel and beryllium moderator in adjacent I-positions for an estimated $10-15 \%$ thermal neutron flux increase [2]. To confirm the neutronic modeling of the effects of the booster fuel and beryllium moderator, a mock-up test was performed at the ATRC reactor with a neutron sensor package deployed at the corresponding ATRC medium I-position with two identical irradiations - with and without the booster fuel and beryllium moderator installed.

Two custom-designed Rh-SPNDs procured from Idaho Laboratories Corporation were installed in the sensor package. The Rh-SPNDs were designed to produce reliably measurable signals during the ATRC medium I-position campaign based on prior experiments at ATRC [3]. Co-located dosimetry was also deployed to provide standardized neutron flux measurements of the experiment.

Data acquisition functionality and sensor performance testing of the Rh-SPNDs prior to the ATRC irradiation were conducted at two other reactors:

1. The AGN-201m reactor at Idaho State University (ISU), which provided neutron flux levels prototypic of the ATRC reactor at $\sim 2 \mathrm{E} 8 \mathrm{n} / \mathrm{cm}^{2}-\mathrm{sec}$ at full power.

2. The Neutron Radiography (NRAD) reactor at Idaho National Laboratory (INL), which allowed performance characterization at an extended range - up to five decades.

Data obtained from the ATRC medium I-loop campaign is used to calibrate the SPNDs performance and experimentally compare against modeled sensitivity predictions.

\section{BACKGROUND}

\subsection{SPND DESCRIPTION}

\subsubsection{Physical Construction}

The standard SPND design uses concentric geometries with the emitter and lead wires encapsulated within a metal sheath filled with mineral insulation (Figure 1). The neutron sensitive region contains the emitter that is responsible for generating the electrical current when the sensor is in a neutron field. The neutron sensitive region transitions to a two-wired mineral-insulated cable (MIC). One lead wire of the MIC attaches to the emitter - called the emitter wire. The other lead wire remains disconnected - called the compensation wire. The SPND design specifications for the ATRC campaign are given in Table 1.

Six total Rh-SPNDs (three of each design) were fabricated and procured on June 26, 2020 as a part of the Advanced Sensor and Instrumentation direct funded program level 3 milestone (M3CT-

20IN070702) - Fabricate Self Powered Neutron Detectors for steady-state reactor operations (Rh-SPND). 
Table 1. SPND materials and geometry comparison between historic Rh-SPND and newly fabricated RhSPNDs from Idaho Laboratories Corporation.

\begin{tabular}{llll}
\hline SPND Design & Emitter & Insulator at NS region & Sheath \\
\hline Large Rh-SPND & Rhodium & $\mathrm{Al}_{2} \mathrm{O}_{3}$ & $\mathrm{I}-600$ \\
(ILC-102-RhSPND) & 0.032 in. OD & 0.072 in. OD & 0.102 in. OD \\
& 3.50 in. L & 0.032 in. ID & 0.072 in. ID \\
Small Rh-SPND & Rhodium & $\mathrm{MgO}$ & $\mathrm{I}-600$ \\
(ILC-080-RhSPND) & 0.020 in. OD & 0.056 in. OD & 0.080 in. OD \\
& 3.50 in. L & 0.020 in. ID & 0.056 in. ID \\
\hline
\end{tabular}

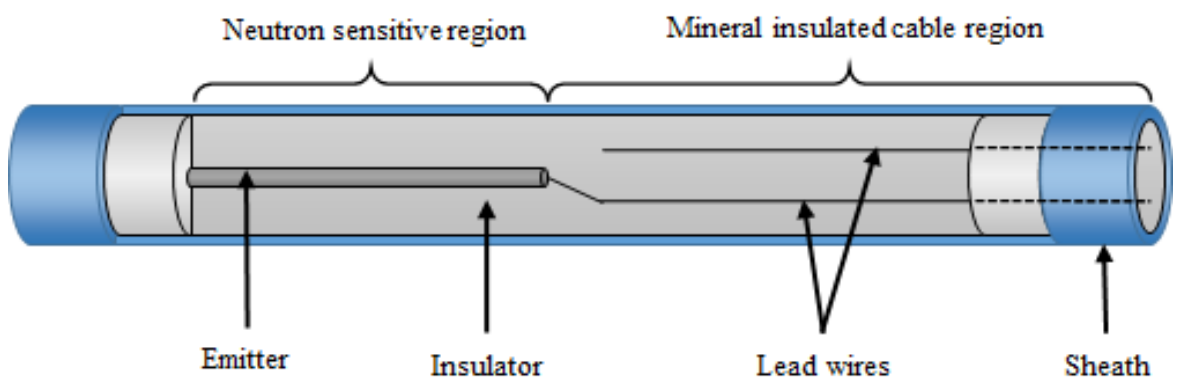

Figure 1. Conceptual view of a standard SPND design.

\subsubsection{Theory and Operation}

As implied by its name, SPNDs are sensors that generate an electrical current in the presence of a neutron field without needing an external power supply. The simplified theory and operation of an SPND is:

1. Neutron interacts with the emitter which results in an ejected electron.

2. The electron transports out of the emitter and deposits in the insulator or sheath generating an electrical current.

3. The electrical current is measured between the emitter wire and the sheath via an electrometer.

However, neutron (and gamma particles) can interact with any of the components including the MIC region of the SPND to eject an electron [4]. Therefore, the process further includes the following:

4. The electrical current is also measured between the compensation wire and the sheath. This represents the contribution to and SPND signal from neutron interactions in the MIC region.

5. The compensation wire current is subtracted from the emitter wire current. The resultant represents the electrical current generation within the neutron sensitive region only.

SPNDs have two different signal characteristics due to the different types of neutron interaction with the sensor. The two characteristics are categorized by their response time- prompt-response and delayedresponse.

Prompt-response signals are generated in SPNDs through gamma interactions within the sensor. Gamma-particles that contribute to generating signals in an SPND can originate from either the sensor or from the reactor environment. While this effect is present in all SPNDs, SPNDs specifically built for prompt-response operation are designed to ensure that gamma particles are preferentially generated in the emitter by using emitter materials with a high neutron capture (and gamma scattering) cross section such as gadolinium, hafnium, or cobalt [5]. 
In addition to prompt-response signals, delayed-response signals are also generated from beta-particle decay within the sensor following neutron capture. The generation of current electrons from this process is therefore characterized by the decay half-life of the materials. As such, materials with a moderately high neutron capture cross section with short activation half-lives, such as rhodium and vanadium, are often used as emitters for SPNDs designed to optimize a delayed-response operation.

Given the short mean free path of beta-particles within the reactor environment, only beta-particles generated within the sensor contribute to the detector signal; as a result, delayed-response SPND signals more accurately reflect the neutron flux and are preferentially chosen over prompt-response optimized SPNDs when applicable such as steady-state operations. To support the medium I-position experimental campaign at ATRC, Rh-SPND was chosen given the steady-state nature of the reactor.

\subsubsection{Delayed-Response Compensation}

General applications of delayed-response SPNDs are for performing flux measurements during steady-state operations where the SPND output can stabilize. However, reactor operations always include power changes from start-up/shutdown, power ramps, and even steady-state perturbations. Therefore, signal compensation techniques need to be applied to provide a more accurate flux trace. Since the response times are well characterized by the activation decay half-lives of the emitter, compensation techniques can be applied.

The delayed-response compensation technique demonstrated in this report is from rearranging the characteristic neutron activation decay Equation (1), and substituting measured data to generate a compensated trace, Equation (2):

$$
\frac{d N_{B}}{d t}=N_{A} \sigma_{A} \varphi(t)-\lambda_{B} N_{B}(t)
$$

Compensated trace is:

$$
i_{c o m p}(t)=\left(\frac{1}{\lambda_{B}} \frac{d i(t)}{d t}+i(t)\right)
$$

Where $\mathrm{i}(\mathrm{t})$ is the measured signal current of the SPND, $\mathrm{di}(\mathrm{t}) / \mathrm{dt}$ is approximated by the symmetric difference quotient, and $\lambda_{\mathrm{B}}$ is the activation product decay constant.

\subsubsection{Preliminary MCNP Modeling}

MCNP6.2 [6] was used to model the sensitivity of the Rh-SPNDs in ATRC. The preliminary model was built as a combination of the two different SPND models [7] [8]. The model used in this work are as follows:

- The SPND neutron sensitive region, as given by Table 1, was expressed in concentric cylinders, spanning only the length defined by the emitters.

- Neutrons were defined as:

- An equally distributed surface source along the outer surface area of the sheath

- A directional distribution of $2 \pi$-inward-normal to the surface area

- An energy distribution, as per previous MCNP analysis of the ATRC configuration provided by the subcontractor, MPR Associates.

- MCNP was set to track neutrons, photons, and electrons under all possible interactions within the modeled region. Additionally, delayed particles from non-fission neutron absorptions were turned on to track beta-particles from neutron activation [9]. 
- An additional run without delayed particles were added to evaluate the model's accuracy for prompt-to-delayed signal values reported elsewhere [7].

- The surface current (F1) tally was used across the surface of the emitter with bins set for tracking electrons moving in and out.

Tallies from the model were converted into neutron sensitivity (A/total flux) as given by Equation (3). The sensitivity was then compared with experimental results - calculated from dividing the actual SPND signal by the measured total flux reported from the collocated dosimetry.

$$
\text { Sensitivity }\left(\frac{\text { Amps }}{\text { total flux }}\right)=(F 1: e(\mu>0)-F 1: e(\mu<0)) * \frac{\left(1.602 \times 10^{-19}\right)}{2 \times S . A .}
$$

Where $\mathrm{F} 1: \mathrm{e}(\mu>0)$ is the number of electrons leaving the emitter, $\mathrm{F} 1: \mathrm{e}(\mu<0)$ is the number of electrons entering the emitter, and S.A. is the outer surface area of the sheath (in $\left.\mathrm{cm}^{2}\right)$.

\section{EXPERIMENTAL SETUP}

The Rh-SPNDs were irradiated in three different reactors with varying conditions and setups with different objectives. The following subsections describe the general setup and objectives of each irradiation:

1. (Performed at INL) Low-to-high $(2.5 \mathrm{~W}$-to-250 kW) power step change at the NRAD reactor incore dry-tube.

2. (Performed at ISU) $5 \mathrm{~W}$ steady-state operation with a prompt-response hafnium-based SPND in the AGN-201m reactor core center.

3. (Performed at INL) $600 \mathrm{~W}$ steady-state operation with collocated dosimetry in the ATRC reactor.

\subsection{Data Acquisition System}

The data acquisition system (DAS) for the SPNDs is composed of a Keithley electrometer with a multiplexing low current scanner card for reading the SPND currents and a National Instruments module coupled to a laptop for data logging. Figure 2 shows the connection sequence from the SPND to the acquisition computerFigure 2. The laptop uses LabVIEW software, shown in Figure 3, to interface with the electronics to collect and record data. Data is recorded sequentially between the individual SPND outputs with a 2,500 ms settling time as required by the electrometer to stabilize between channels of the scanner card.

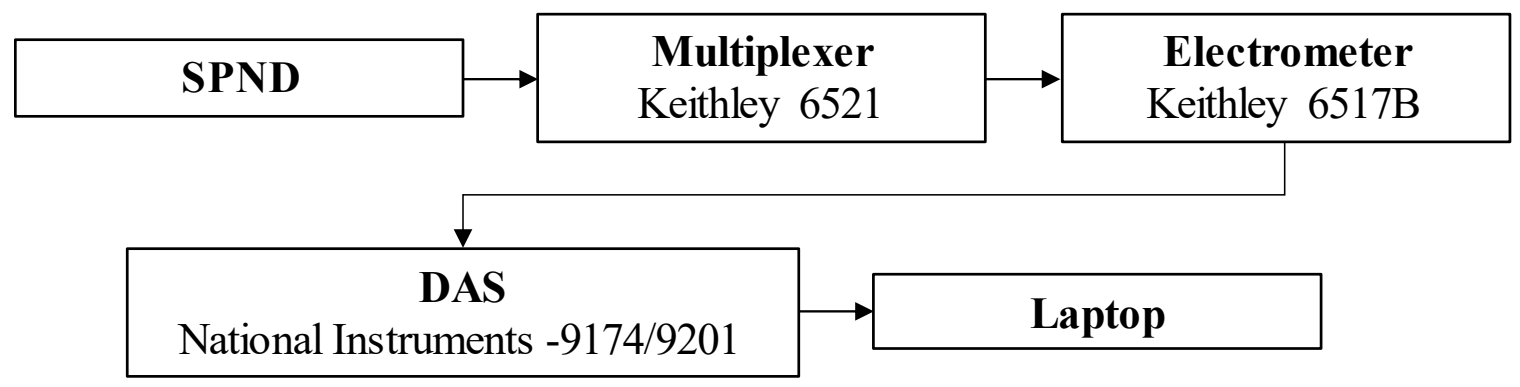

Figure 2. SPND data acquisition configuration. 


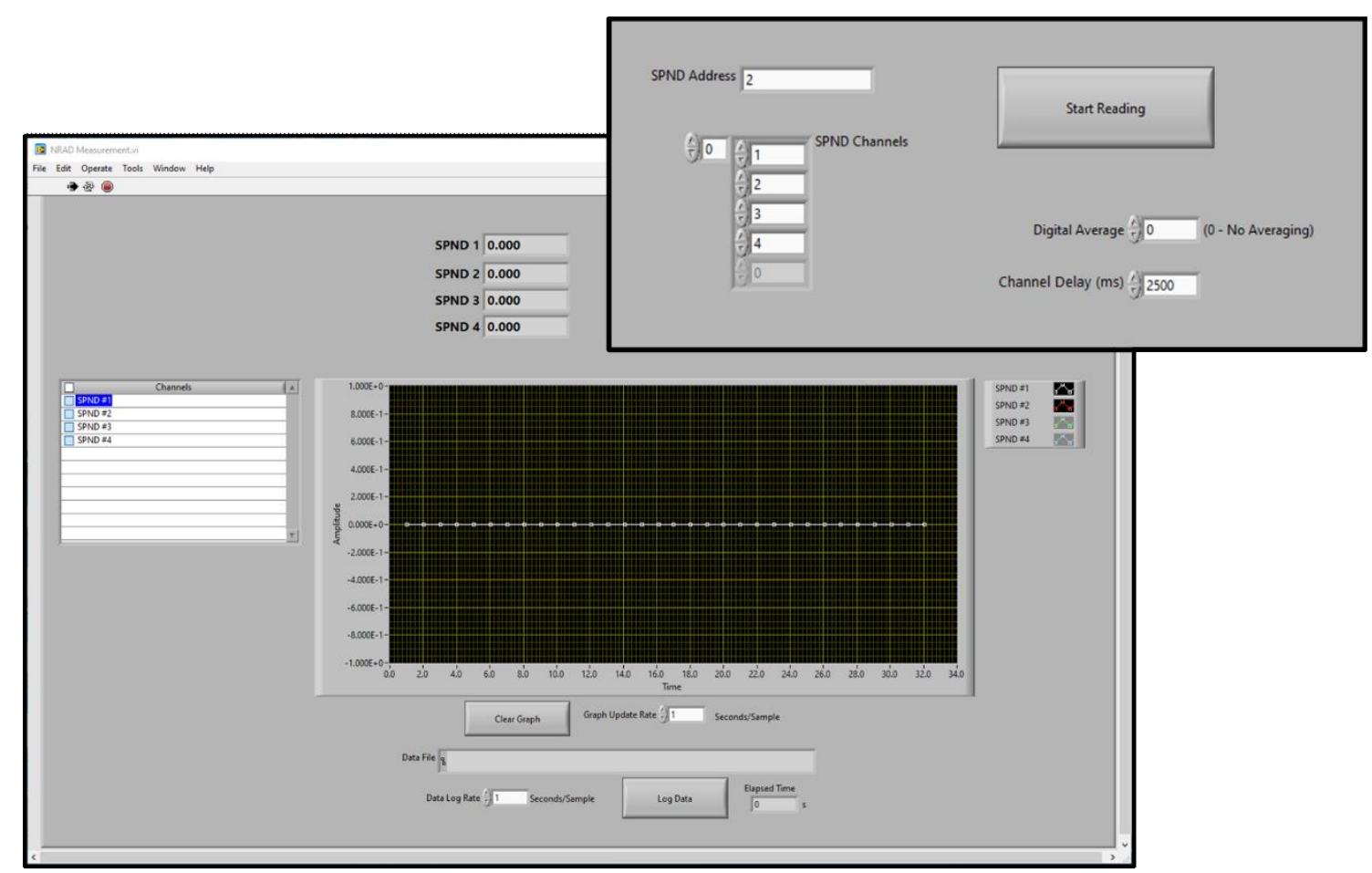

Figure 3. LabVIEW software interface for SPND data recording.

\subsection{Sensor Deployment}

\subsubsection{SPND Low-Flux Resolution Testing at AGN-201m}

The AGN-201m reactor at ISU is a $5 \mathrm{~W}$ polyethylene moderated research reactor with an open centerline port that penetrates through the center of the core for experimental use (Figure 4). At full power, the estimated thermal neutron flux in the core center penetration is estimated to be $\sim 2 \mathrm{E} 8 \mathrm{n} / \mathrm{cm}^{2}$ sec.

The main objective of the SPND irradiation at the AGN-201m reactor is to test the Rh-SPND and electronics signal resolution at the low-flux level. A second objective is to test the delayed-compensation technique. A sensor bundle comprised of a dedicated large Rh-SPND (ILC-102-RhSPND) and a hafniumbased SPND was inserted into the AGN-201m centerline port with the emitters positioned at the axial center of the core. Data from the two SPNDs were recorded within the same DAS as power was ramped to $5 \mathrm{~W}$ and maintained power for approximately 35 minutes. The Rh-SPND signal trace, both uncompensated and compensated, were compared to the Hf-SPND signal. 


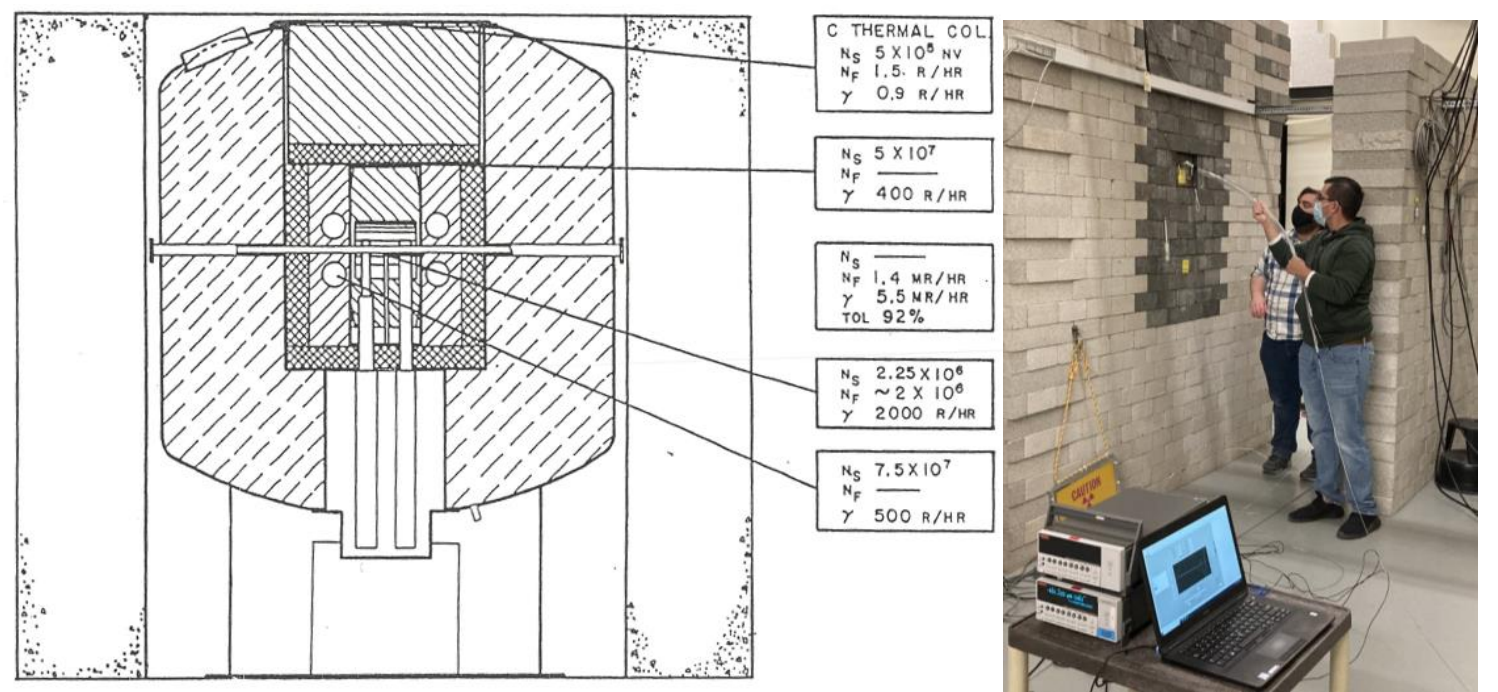

Figure 4. (Left) AGN-201m cross-sectional diagram with radiation levels of the reactor operating at $5 \mathrm{~W}$. (Right) Insertion of Rh-SPND and Hf-SPND into the core center of AGN-201m reactor.

\subsubsection{SPND Functional Range Testing at NRAD}

The NRAD) reactor at INL is a $250 \mathrm{~kW}$ pool-type (TRIGA) reactor primarily used for neutron radiography. However, the reactor has two irradiation positions - the one at position F-1, shown in Figure 5, is an easy-access dry-tube. The neutron flux at the fuel mid-axial plane within the F-1 dry-tube is relatively constant at $2.1 \mathrm{E} 9 \mathrm{n} / \mathrm{cm}^{2}-\mathrm{sec}$ at $250 \mathrm{~W}$ (Figure 6).

The main objectives of SPND irradiations at NRAD is to measure sensor-electronics noise and verify SPND performance at an extended range - up to six decades. A dedicated small Rh-SPND (ILC-080RhSPND) was inserted into the F-1 dry-tube and positioned such that the emitter is centered at the fuel mid-axial plane, and two irradiations were performed:

- $25 \mathrm{~W}$ to $25 \mathrm{~kW}$ power step change per decade of reactor power (neutron flux ranges from $\sim 2 \mathrm{E} 8$ to $\left.\sim 2 \mathrm{E} 11 \mathrm{n} / \mathrm{cm}^{2}-\mathrm{sec}\right)$.

- $\quad 2.5 \mathrm{~W}$ to $250 \mathrm{~kW}$ power step change per decade of reactor power (neutron flux ranges from $\sim 2 \mathrm{E} 7$ to $\sim 2 \mathrm{E} 12 \mathrm{n} / \mathrm{cm}^{2}-\mathrm{sec}$ ).

The recorded Rh-SPND signal traces, both uncompensated and compensated, were then compared to reactor power data to evaluate the compensation technique and investigate sensor linearity. 

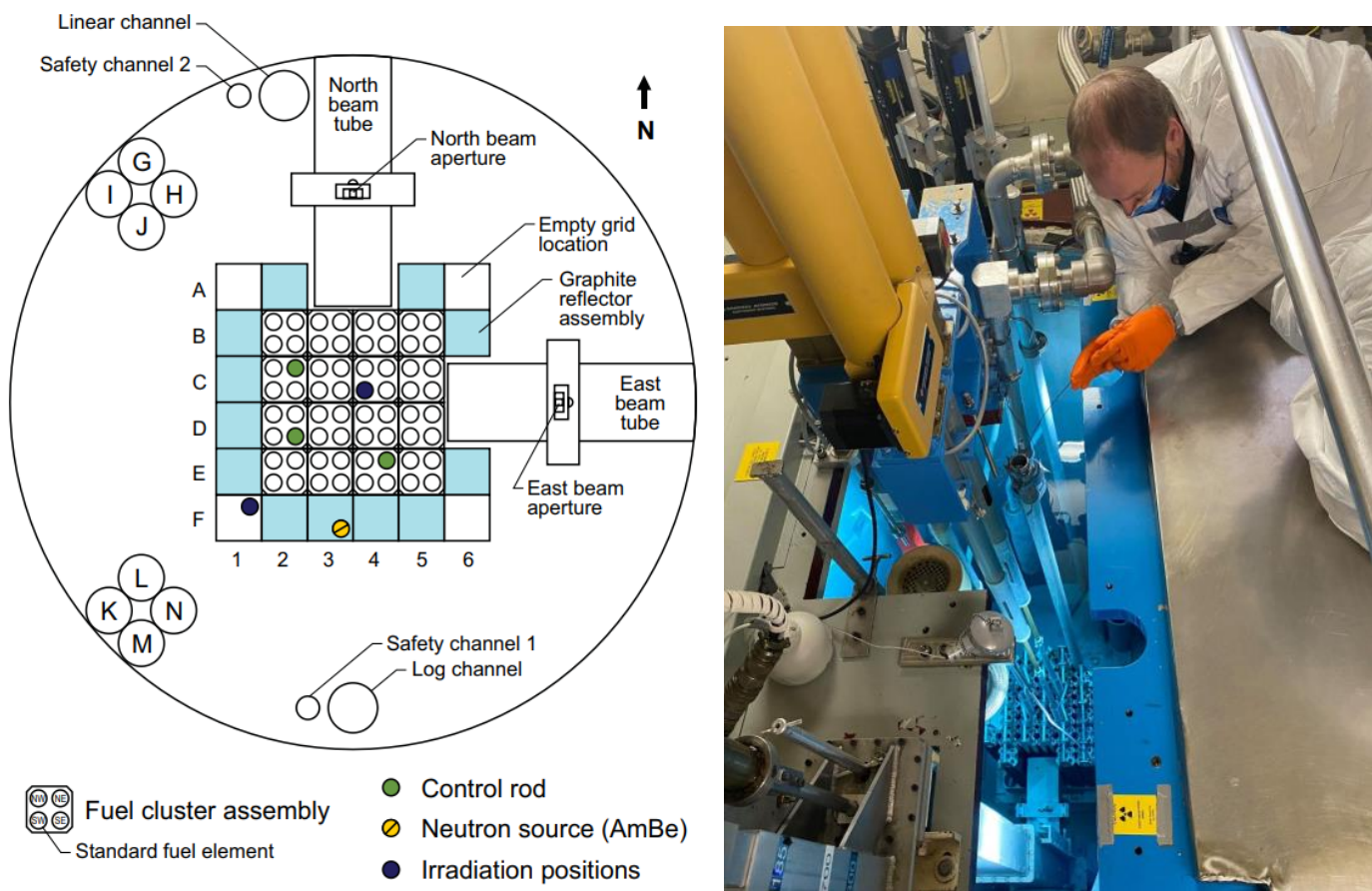

Figure 5. (Left) NRAD core configuration. (Right) Insertion of Rh-SPND into the F1 irradiation position.

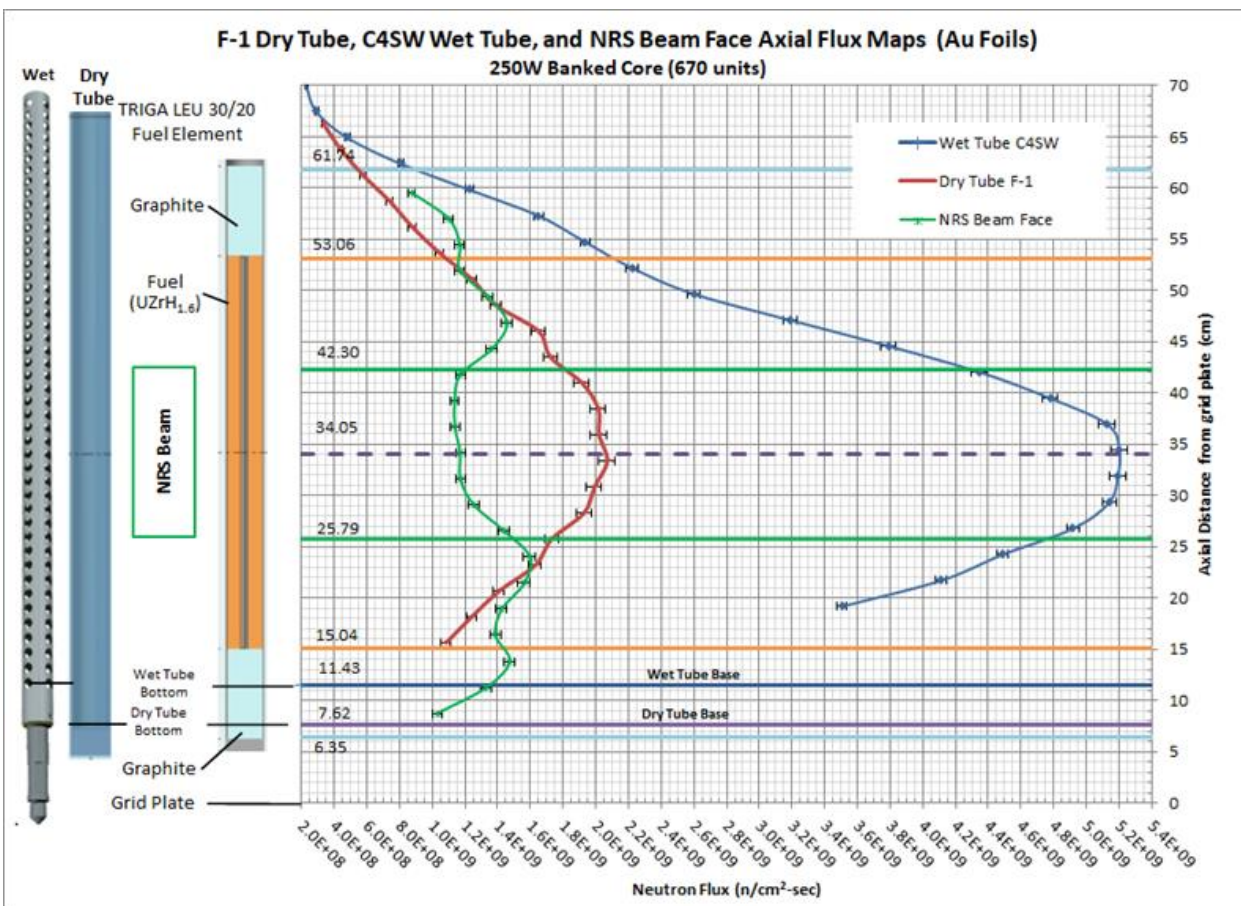

Figure 6. NRAD axial neutron flux distribution at $250 \mathrm{~W}$.

\subsubsection{Rh-SPND Deployment for ATRC Medium I-Position Campaign}

The ATRC reactor is a 5kW-licensed pool-type reactor built as an approximate full-scale nuclear mock-up of the ATR core. The differences between the ATRC and ATR core configurations are due to upgrades performed to ATR that were not duplicated in the ATRC. However, the differences are 
considered insignificant enough such that ATRC is still used to experimentally confirm predicted reactivity changes prior to carrying out the actual experiment in ATR.

The ATRC medium I-position experiment campaign occurred in the southern I-positions of the ATRC. Both designs of the Rh-SPND were inserted into the sensor package. The sensor package is positioned in the western-most medium I-position for both irradiations (Figure 7):

- Steady-state operation at $600 \mathrm{~W}$ for 6 hours without the beryllium moderator and booster fuel.

- Steady-state operation at $600 \mathrm{~W}$ for 6 hours with the beryllium moderator and booster fuel.

The data from the Rh-SPNDs were both recorded within the same DAS. Sensor traces were then compared with the reactor power log.

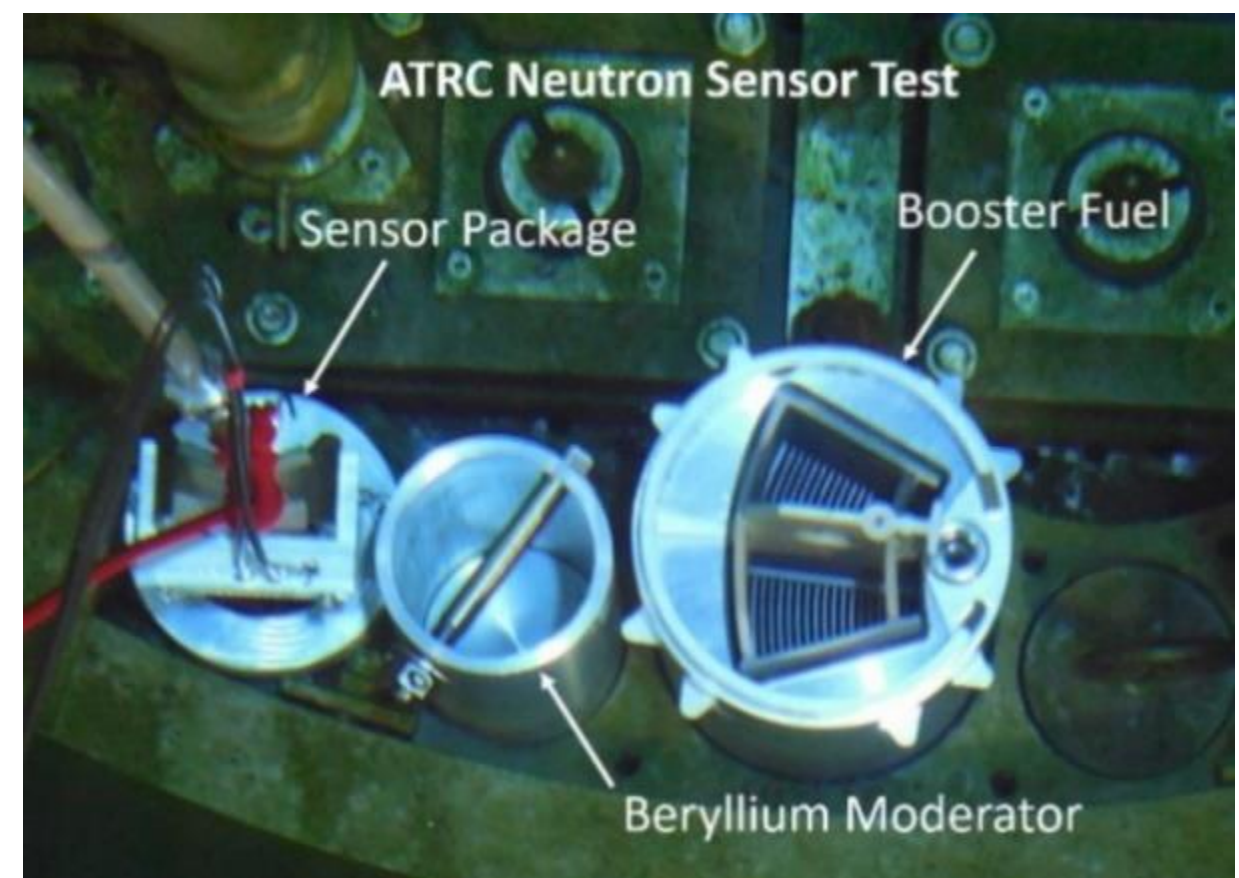

Figure 7. Conceptual image of the booster fuel and beryllium moderator deployed in ATR/ATRC.

\section{RESULTS}

\subsection{SPND Signal Comparisons}

\subsubsection{SPND Performance in AGN-201m}

The overlayed signal trace of the large Rh-SPND with the Hf-SPND is shown in Figure 8. The delayed-signal compensation technique from Equation (2) is applied to the Rh-SPND signal trace to demonstrate the method's capability, as shown by the signal rise and fall response at the 10 minute and 50 minute mark. 

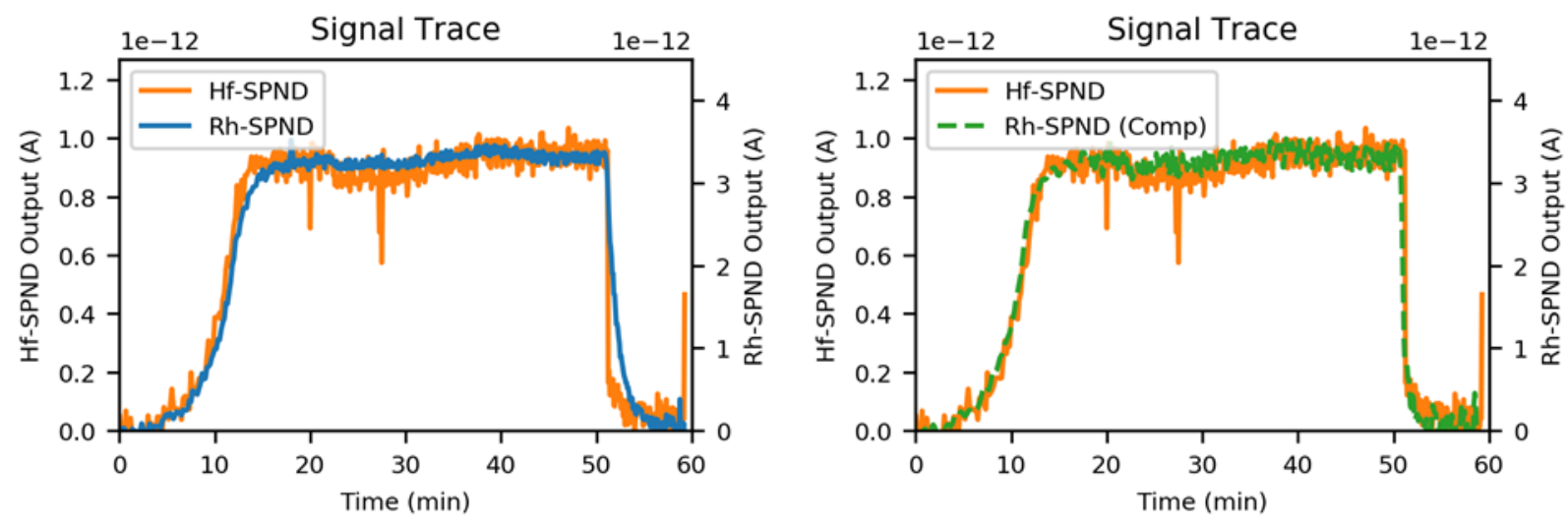

Figure 8. Signal comparison of (left) uncompensated and (right) compensated Rh-SPND with Hf-SPND.

\subsubsection{SPND Performance in NRAD}

The overlayed signal trace of the small Rh-SPND with reactor power is given by Figure 9. A linearity comparison between the compensated Rh-SPND signal to reactor power was performed. The plot is given in Figure 10. The result of the linear regression is given in Equation (4).

Signal Trace

Performance at NRAD 11/3/2021

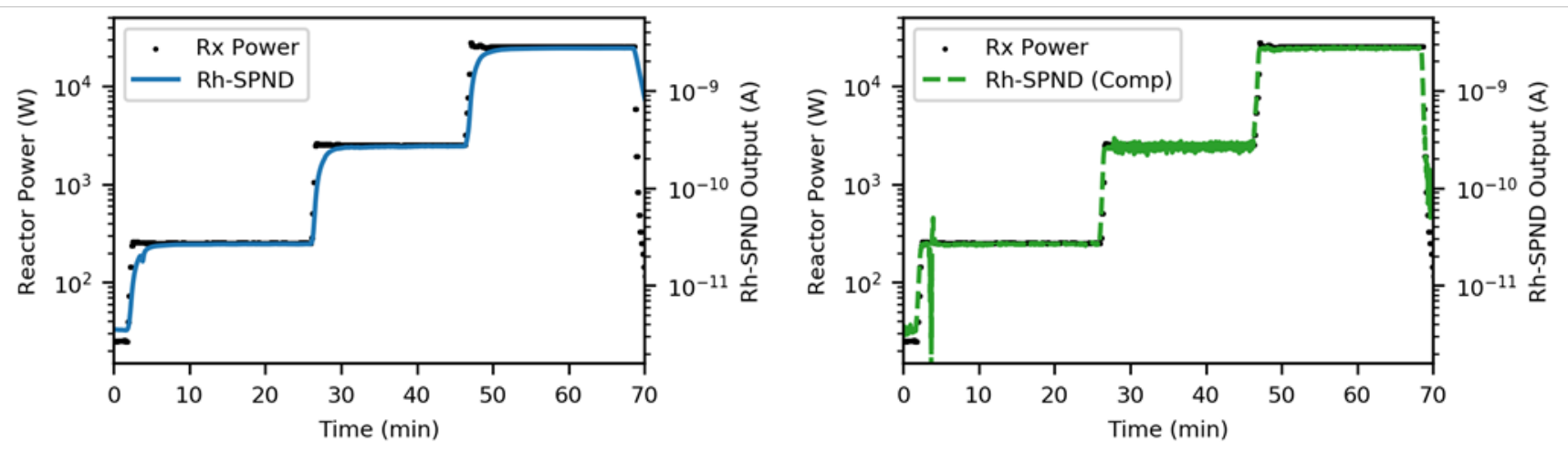

Performance at NRAD 12/8/2021
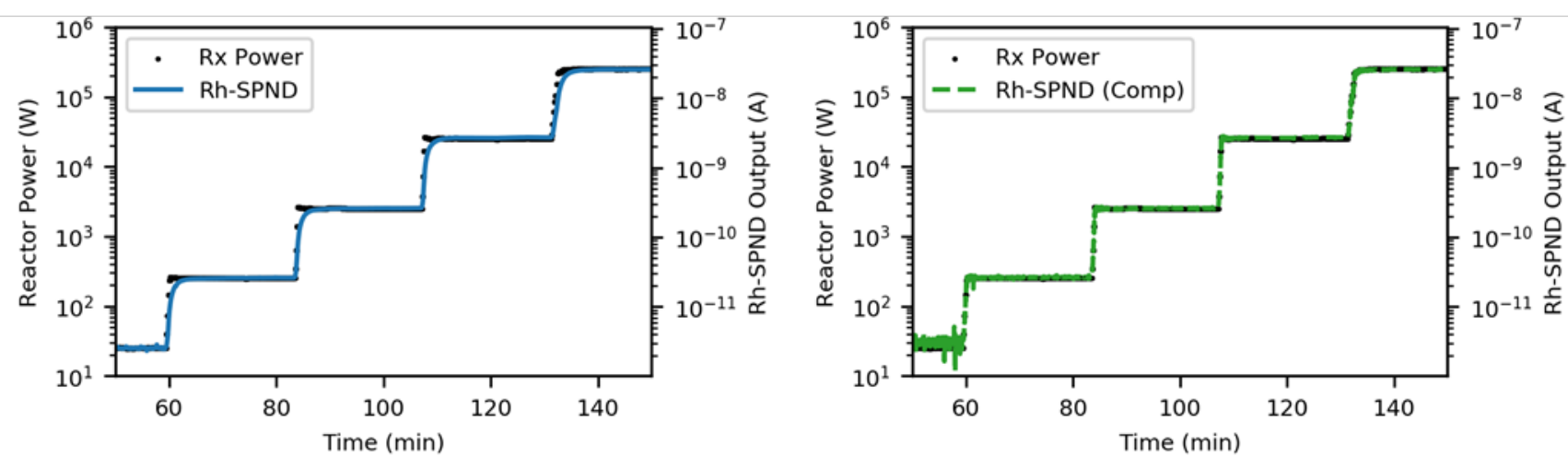

Figure 9. Signal comparison of (left) uncompensated and (right) compensated Rh-SPND with NRAD power. 


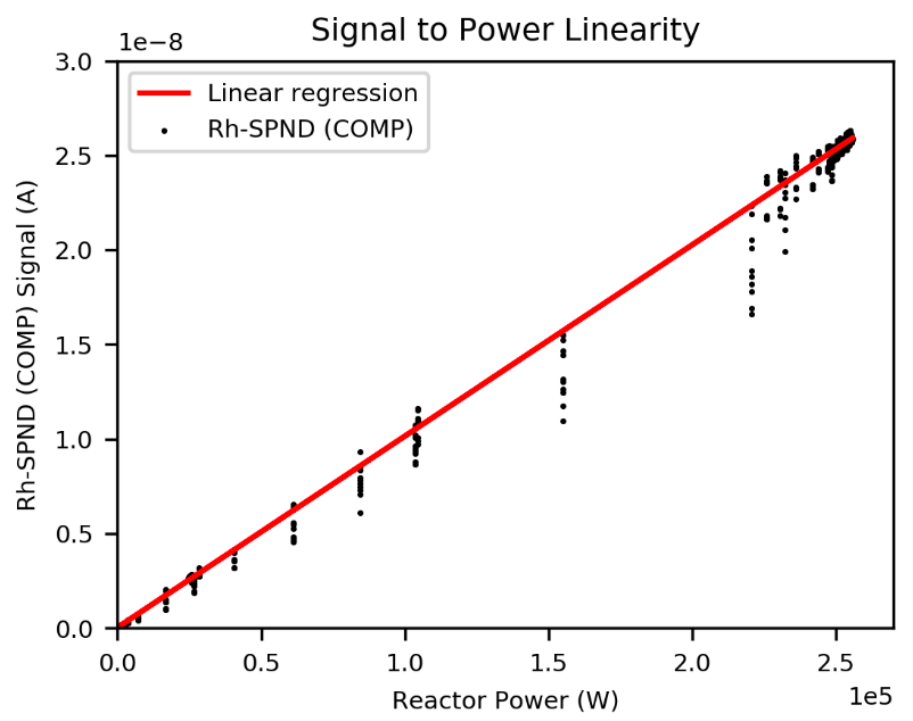

Figure 10. Rh-SPND signal linearity plot.

$$
\begin{gathered}
\operatorname{Signal}(A)=1.0129 \times 10^{-13}\left(\frac{A}{W}\right) \times \operatorname{Power}(W)+2.6469 \times 10^{-11}(A) \\
r^{2}=0.9927
\end{gathered}
$$

\subsubsection{SPND Performance in ATRC}

Final deployment of the Rh-SPNDs was in the sensor package inserted into the medium I-position of the ATRC reactor. The signal trace from both Rh-SPNDs designs during the two-consecutive irradiations without and with the fuel booster and beryllium moderator is shown in Figure 11 and Figure 12.

The relative difference in SPND outputs between the runs is compared with dosimetry results and is tabulated in Table 2 . 
Signal Trace

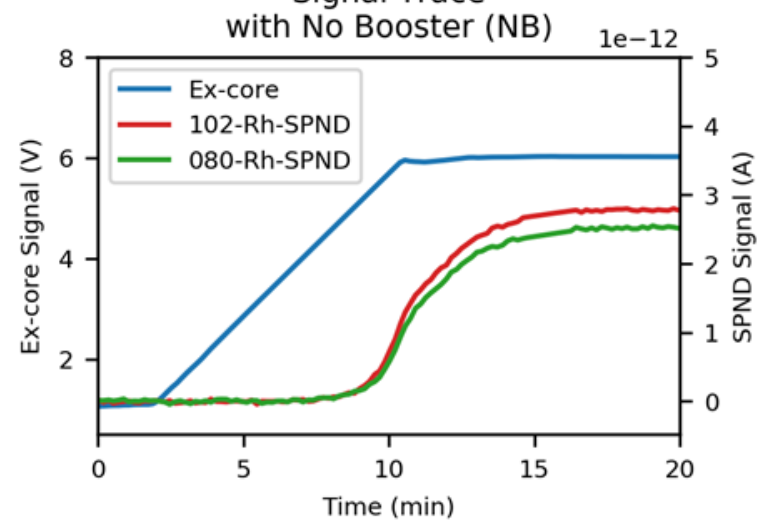

Signal Trace

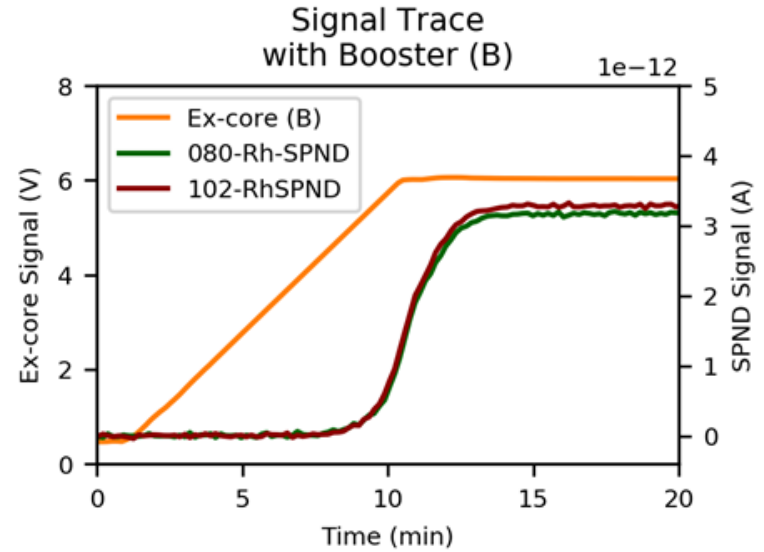

Signal Trace

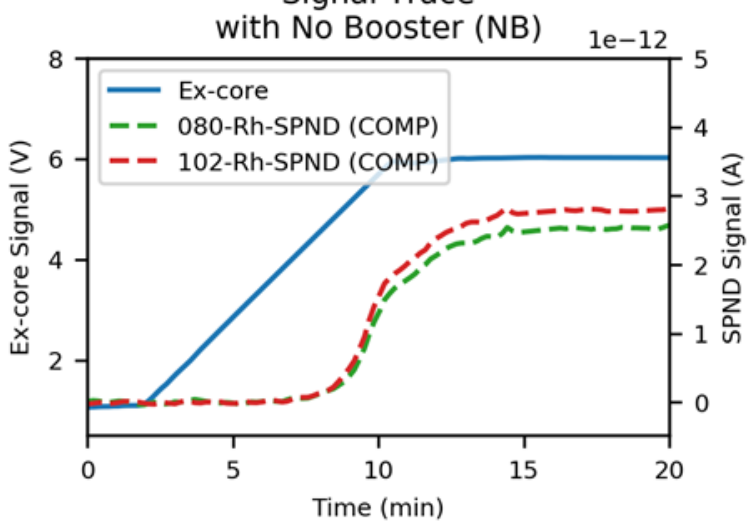

Signal Trace

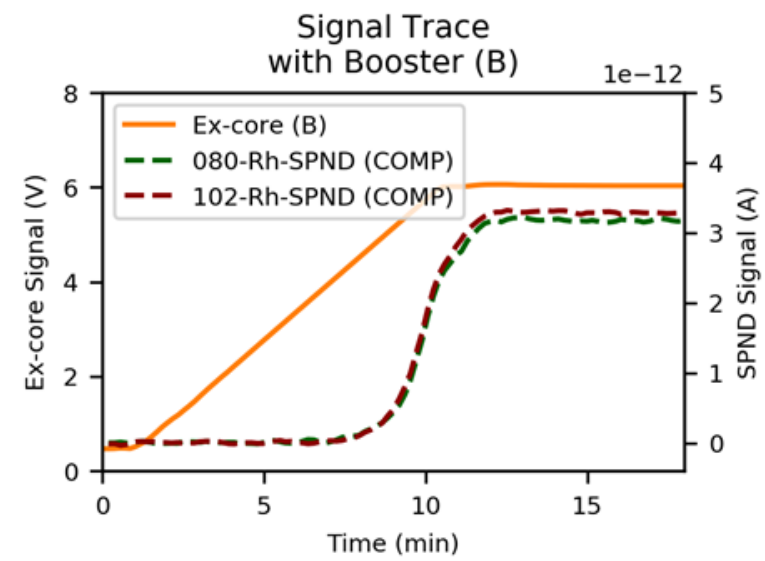

Figure 11. Signal Trace of 080-Rh-SPND and 102-Rh-SPND during ATRC irradiations.
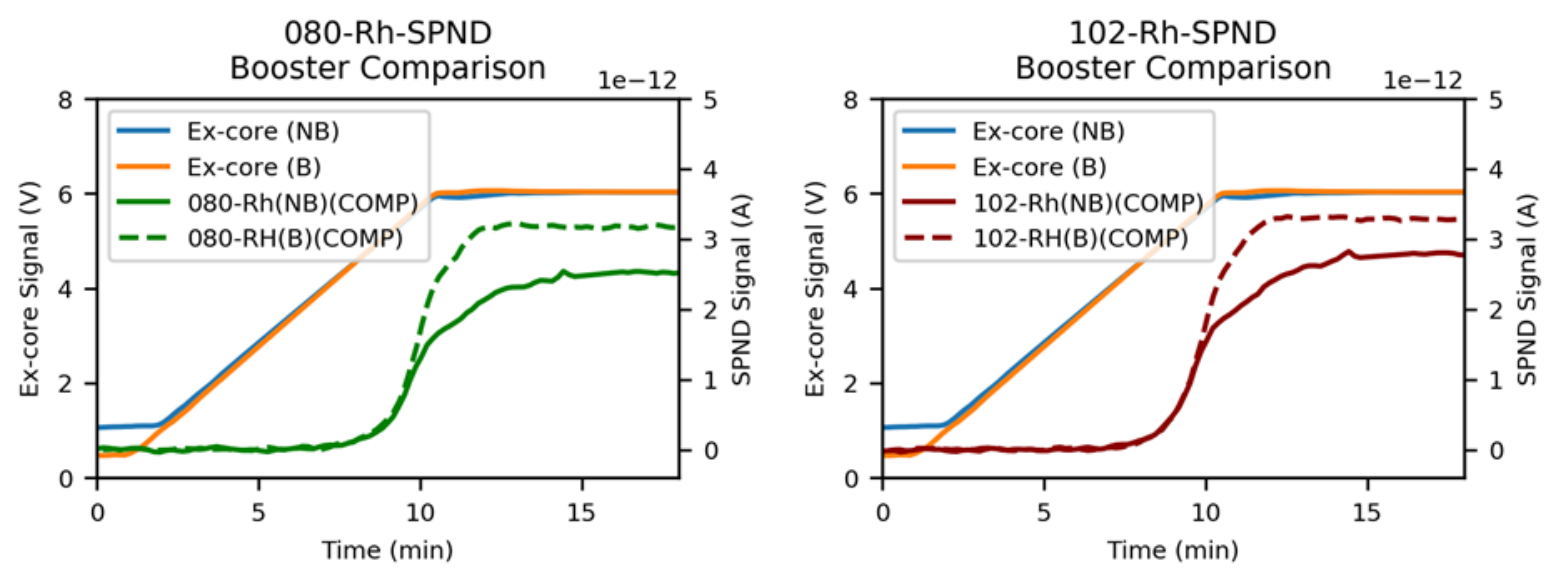

Figure 12. Comparison of individual Rh-SPND output between irradiations with no booster inserted (NB) and booster inserted (B). 
Table 2. Experimental measurement comparison between irradiations without and with booster fuel and moderator.

\begin{tabular}{cccc}
\hline ATRC Irradiation & 080-SPND & 102-Rh-SPND & Co Axial Flux wire \\
\hline $\begin{array}{c}\text { Without booster fuel } \\
\text { and moderator (NB) }\end{array}$ & $2.5018 \pm 0.0246 \mathrm{pA}$ & $2.7343 \pm 0.0173 \mathrm{pA}$ & $4.377 \pm 0.175 \mathrm{E} 8 \phi_{\mathrm{th}}$ \\
$\begin{array}{c}\text { With booster fuel and } \\
\text { moderator (B) }\end{array}$ & $3.1555 \pm 0.0222 \mathrm{pA}$ & $3.2290 \pm 0.0212 \mathrm{pA}$ & $5.341 \pm 0.204 \mathrm{E} 8 \phi_{\mathrm{th}}$ \\
$\begin{array}{c}\text { Relative measurement } \\
\text { increase }\end{array}$ & $1.2613 \pm 0.0153$ & $1.1809 \pm 0.0108$ & $1.218 \pm 0.047$ \\
\hline
\end{tabular}

\subsection{MCNP Sensitivity Comparison}

In addition to performing measurements, the SPND sensitivities were calculated as a part of signal strength prediction during the design phase for the SPNDs. As described in Section 2.1.2.2, the results of the MCNP preliminary sensitivity prediction were compared with experimental measurements. The results are tabulated in Table 3.

Table 3. Comparison between experimental peak signal vs. MCNP prediction.

\begin{tabular}{cccc}
\hline & & & Relative \\
& 080-SPND & 102-Rh-SPND & 080-SPND/102-SPND \\
\hline Experimental & $2.5018 \pm 0.0246 \mathrm{pA}$ & $2.7343 \pm 0.0173 \mathrm{pA}$ & $0.915 \pm 0.030$ \\
MCNP Predicted & $10.28 \pm 0.05 \mathrm{pA}$ & $16.09 \pm 0.08 \mathrm{pA}$ & $0.639 \pm 0.094$ \\
\hline
\end{tabular}

\section{CONCLUSION}

Two designs of custom-made Rh-SPNDs were procured from Idaho Laboratories Corporation for the purpose of performance demonstration, calibration, and qualification in steady-state reactor operations. Secondary to this effort was the deployment of the same design of SPNDs into ATRC in support of the medium I-position campaign.

The Rh-SPNDs were tested in AGN-201m for signal strength to demonstrate a sensor sensitivity capable of supporting deployment in ATRC. Additionally, a prompt-response compensation technique was performed on the Rh-SPND signal trace. The compensated signal was compared with a promptresponse Hf-SPND to demonstrate the technique's accuracy.

The Rh-SPNDs were also tested in the NRAD reactor to verify its performance across a different reactor and to demonstrate the sensor's linearity for up to five decades of reactor power. Sensor linearity performance within the NRAD reactor showed a sensitivity of $1.0129 \times 10^{-13} \mathrm{~A} / \mathrm{W}$ with a linear performance with a correlation-squared value of $r^{2}=0.9927$.

With the full investigation of the SPNDs performance with respect to sensitivity, compensation technique, and linearity, both Rh-SPND designs were deployed in the ATRC reactor. Both SPNDs were able to measure an increased flux $\sim 20 \%$ between the two reactor operations. This measurement fell well between the relative flux increase as measured through collocated conventional dosimetry.

Preliminary MCNP modeling showed an approximately higher predicted signal (and sensitivity) by a factor of 5; however, factors that decrease sensitivity have yet to be taken into account, such as charge 
deposition within the insulator and its correlated electric field to reduce sensitivity as well as background gamma- and beta-particle contributions [5].

Overall, both Rh-SPND designs demonstrated good performance in measuring low-flux steady-state operations; as such, these same sensors will be used in FY-21 and FY-22 to support other planned experiments. However, further investigation into the sensor sensitivity and MCNP modeling will continue as part of the FY-21 activities.

\section{REFERENCES}

[1] INL/MIS-19-55710, "Conceptual Design Report for the I2 Instrumentation Experiment in ATRC," Idaho National Laboratory, 2019.

[2] DOE-ID-INL-19-059 R1, "DOE-ID NEPA CX Determination: I-Loop Deployment in the Advanced Test Reactor," 2020.

[3] INL/EXT-13-29896, "ATRC Neutron Detector Testing Quick Look Report," 2013.

[4] C. J. Allan and G. F. Lynch, "Signals Produced by Inconel Mineral Insulated Coxial Cables in Neutron and Gamma-Ray Fields," Atomic Energy of Canada Limited, Chalk River, Ontario, 1980.

[5] H. D. Warren and N. H. Shah, "Neutron and Gamma-Ray Effects on Self-Powered In-Core Radiation Detectors," Nuclear Science and Engineering, vol. 54:4, pp. 395-415, 1974.

[6] C. J. Werner(editor), "MCNP Users Manual - Code Version 6.2," Los Alamos National Laboratory, 2017.

[7] L. Barbot, V. Radulovic, D. Fourmentel, L. Snoj, M. Tarchalski, V. Dewynter-Marty and F. Malouch, "Calculation to Experiment Comparison of SPND Signals in Various Nuclear Reactor Environments," in ANIMMA 2015 - 4th International Conference on Advancements in Nuclear Instrumentation Measurement Methods and their Applications, Lisbonne, Portugal, 2015.

[8] J. A. King, A. M. Miller and E. J. Parma, "SPND Sensitivity Calculations Using MCNP and Experimental Data from ACRR," Sandia National Laboratories, Alburquerque, New Mexico, 2020.

[9] G. W. McKinney, "MCNP6 Enhancements of Delayed-Particle Production," in IEEE 2012 Nuclear Science Symposium and Medical Imaging Conference, Anaheim, California, 2012.

[10] INL/EXT-08-14709, "Advanced Test Reactor National Scientific User Facility User's Guide," 2009. 